Article

\title{
On the Micromixing Behavior of a Spinning Disk Reactor for Metallic Cu Nanoparticles Production
}

\author{
Angela Marchetti and Marco Stoller *(1) \\ Department of Chemical Materials Environmental Engineering, Sapienza University of Rome, \\ Via Eudossiana 18, 00184 Rome, Italy \\ * Correspondence: marco.stoller@uniroma1.it; Tel.: +39-06-4458-5580
}

Received: 17 June 2019; Accepted: 7 August 2019; Published: 12 August 2019

check for updates

\begin{abstract}
The interest in producing copper nanoparticles is high, mainly due to their enhanced properties, a wide range of possible and different applications, and the possibility for their use in the framework of catalysis. The purpose of this study is to synthesize copper nanoparticles by chemical reduction of copper sulfate with L-ascorbic acid and sodium borohydride capable to eliminate free radicals providing an antioxidant environment promoting pure copper nuclei formation. The micromixing conditions necessary for the nanoparticles production are provided by a spinning disk reactor (SDR). Relevant operational parameters, such as the disk speed velocity and the position of the reactant injectors, will lead to different product outcomes. The latter was checked by means of a dynamic light scattering instrument (DLS). At the end, depending on the adopted operating conditions, the SDR was able to produce particles between $16 \mathrm{~nm}$ and $39 \mathrm{~nm}$, with a particle size distribution (PSD) characterized by a narrow, monomodal plot. In comparison to the smallest particles obtained in a stirred reactor tank, that is, $132 \mathrm{~nm}$, the obtained results appear to be very promising.
\end{abstract}

Keywords: copper nanoparticles; spinning disk reactor; catalyst application

\section{Introduction}

The interest in producing metal nanoparticles is connected to the possibility of employing them in different fields of possible applications with enhanced chemical and physical performances, such as the use in catalysis, in absorption, for chemical and biological sensors, and for photonic and electronic devices [1]. Another advantage is represented by the possibility of using less-noble metals, such as titania or copper, as an alternative to gold or silver with a reduction of relevant material costs. Recently, interest focused on copper nanoparticles production in the framework of the electronic industry, substituting silver in the electroconductive inks, the analytical sensor industry, and aids environmental remediation purposes [2,3]. In addition to this, copper results appear to be attractive for the preparation of $\mathrm{Cu}$-based nanoparticles due to their extensive range of possible oxidation states $\left(\mathrm{Cu}^{0}, \mathrm{Cu}^{\mathrm{I}} \mathrm{Cu}^{\mathrm{II}}\right.$ and $\left.\mathrm{Cu}^{\mathrm{III}}\right)$, promoting different possible chemical reactions. However, there are some disadvantages in the use of $\mathrm{Cu}$, which are mainly connected to the need to protect the particles from oxidation by the environment during storage and the difficulty to reduce copper ions under mild reaction conditions [4]. Minor difficulties are the tendency to agglomerate and the difficulty to synthesize large quantities of high-quality nanomaterial capable of satisfying the industrial needs.

Nowadays, the main method to produce metal nanoparticles is to use stirred reactors in batch. However, this method is not capable of producing the growing amounts required by the industry. An alternative to obtain copper nanoparticles is represented by the use of some physical methods, such as thermal decomposition, capable of producing larger quantities in the form of nanopowder but characterized by low purity levels [5]. As a consequence, a great interest by the relevant industry to develop novel routes to produce high quantities of copper nanoparticles of high quality remains [6]. 
A promising approach to satisfy these requirements appears to follow the route of the wet chemical synthesis that allows continuous production by employing process intensified equipment with limited investment and operational costs. Possible alternatives are continuous hydrothermal reactors, continuous microwave reactors, tubular flow reactors, T-mixers, and the spinning disk reactor [7]. Each equipment exhibits different capabilities to produce the copper nanoparticles in size and shape. In this work, a spinning disk reactor (SDR) was used at different operating conditions by varying the disk velocity, the number and position of the reactants injectors, and the reactant flow rates to study the reaction outcome of copper nanoparticles production in terms of size and purity, and to check if this equipment is capable to reach the requirement targets of the industry. Indeed, the advantages of the SDR are low investment and operations costs compared to other technologies, continuous and high production capacities, and the ease of use of the equipment [8]. Beforehand, the technology was employed for the production of many other materials, such as titania, hydroxyapatite, zero valent iron, and bi-metal nanoparticles [9-11].

\section{Materials and Methods}

\subsection{Materials}

To produce the copper nanoparticles, aqueous solution of copper sulfate pentahydrate $\mathrm{CuSO}_{4} \cdot 5 \mathrm{H}_{2} \mathrm{O}$ (Sinopharm Chemical Reagent Co., Ltd., Shanghai, China) was mixed separately with L-ascorbic acid $\left(\mathrm{C}_{6} \mathrm{H}_{8} \mathrm{O}_{6<}\right.$ Sinopharm Chemical Reagent Co., Ltd., Shanghai, China) and sodium borohydride $\left(\mathrm{NaBH}_{4}\right.$-Reagent Plus 99\%, Sigma-Aldrich, St. Louis, MO, USA), both reducing reagents and capping agent.

\subsection{Synthesis of Copper Nanoparticles}

$\mathrm{CuSO}_{4} \cdot 5 \mathrm{H}_{2} \mathrm{O}$ aqueous solution was prepared by dissolving $\mathrm{CuSO}_{4} \cdot 5 \mathrm{H}_{2} \mathrm{O}(10 \mathrm{mmol})$ in $50 \mathrm{~mL}$ deionized water in a stirred flask heated up to $80^{\circ} \mathrm{C}$. The resulting solution was then mixed to a stochiometric concentrated aqueous solution of L-ascorbic acid. In case of the stirred tank reactor, the first reagent was poured into a $200 \mathrm{~mL}$ Becher glass, whereas the second reagent was added drop-wise providing mixing by a magnetic propeller. In case of the SDR, the two solutions were injected simultaneously. In both cases, the reaction outcome is observed as a dark solution. The resulting dispersion was centrifuged at $8000 \mathrm{rpm}$ for $15 \mathrm{~min}$. The supernatant was placed under ambient conditions for two months. The same procedure was adopted when sodium borohydride was used.

\subsection{Characterization}

To characterize the synthesized copper nanoparticles, a nanosizer based on DLS was used (Brookhaven Plus 90). The measuring principle of this device relays on the resolution of an auto-, leading to the determination of a particle size distribution profile (PSD).

\subsection{Methods}

The adopted method in this work to produce the copper nanoparticles relies on the use of an SDR that allows the operation in wet continuous conditions. Compared to other technologies available to nanoparticle production, the SDR has the advantage to exhibit the required and specific micromixing conditions in the framework of a low energy-consuming, continuous operation, allowing to obtain a high quantity of nanoparticles of specific PSD. This characteristic appears to be compatible to industrial practice [12].

The SDR consists of an external reactor vessel hosting a rotating disc of $8.5 \mathrm{~cm}$ in diameter, on which surface the mixing of the poured reactants and the consequent reaction will take place. The scheme of the adopted SDR is reported in Figure 1. The rotation speed of the disk, $\varpi$, is one of the most important operating parameters that directly affects the micromixing conditions and was changed to values between $500 \mathrm{rpm}$ and $1500 \mathrm{rpm}$ [13]. Together with the reactants flow rate and feeding 
point number and position, it is possible to obtain the nanoparticles of specific narrow, unimodal PSD. For all experiments, the used injectors were of $0.2 \mathrm{~cm}$ in diameter and positioned $2 \mathrm{~cm}$ above the disk surface [14]. The temperature of the solutions was kept constant at $80^{\circ} \mathrm{C}$. The product was continuously removed from the outlet at the bottom of the reactor which was maintained at room temperature.

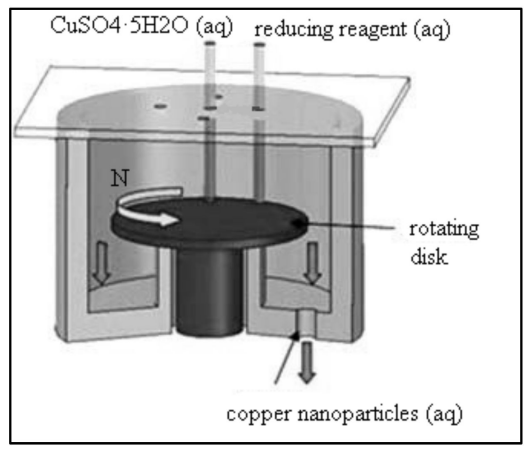

Figure 1. Scheme of the adopted spinning disk rotator (SDR).

The use of the SDR is aimed to promote the mixing among the reagents with low contact times in order to maximize the product concentration with high nucleation rates and very low particle growth. The efficiency of the precipitation reaction depends on the mixing within the system. If the mixing is not adequate, the reagent streams penetrate the aqueous bulk formed on the disk, creating three segregated phases. Due to this stratification, the reaction takes place without the optimal $\mathrm{pH}$, leading to the formation of bigger and unstable particles with a higher tendency to agglomerate. Therefore, the optimal conditions are obtained with a rapid mixing of the streams, low contact times of the reagents, high nucleation rate, and low supersaturation available for the particle growth. All these aspects are favored by a high rotational velocity of the disk in the overall film thickness [8].

\section{Results and Discussion}

\section{Experimental Results}

In the first step, two batch runs were carried out at different stirring rates to check the performances in term of minimum size of produced copper nanoparticles using different reducing agents. The results are reported in Figure 2.

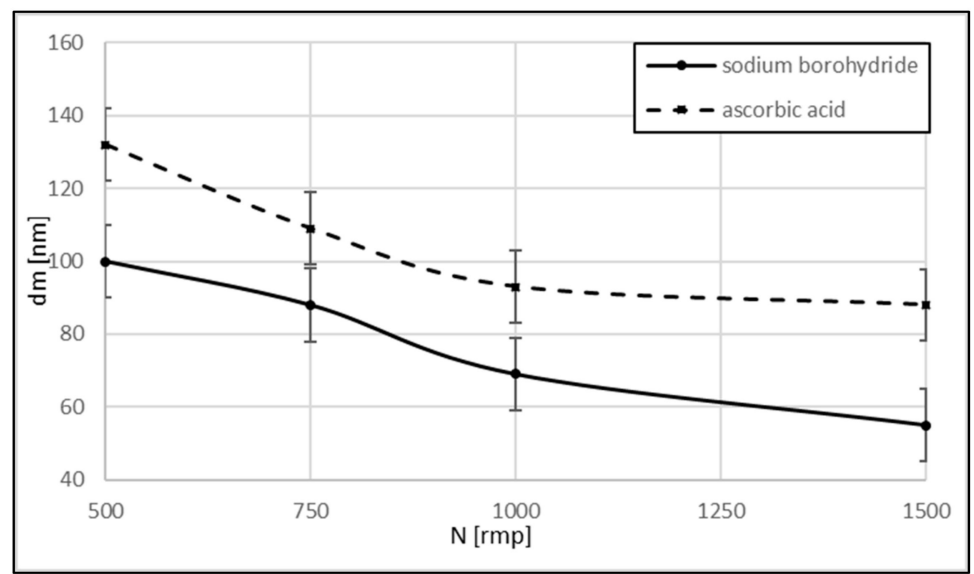

Figure 2. Results obtained by adopting the stirred tank reactor. 
It is possible to observe that the size of the produced copper nanoparticles is smallest when sodium borohydride is used at maximum stirring conditions that are equal to $55 \mathrm{~nm}$. As a consequence, for further tests adopting the SDR, sodium borohydride was selected as reducing agent.

In the second step, the outcome of the reaction, performed by means of the SDR as a function of the disk velocity, was checked. In this work, two different feeding point configurations were used, that is, feeding by two $(\mathrm{nf}=2)$ or four $(\mathrm{nf}=4)$ injection points, respectively. The configurations are reported in Figure 3.

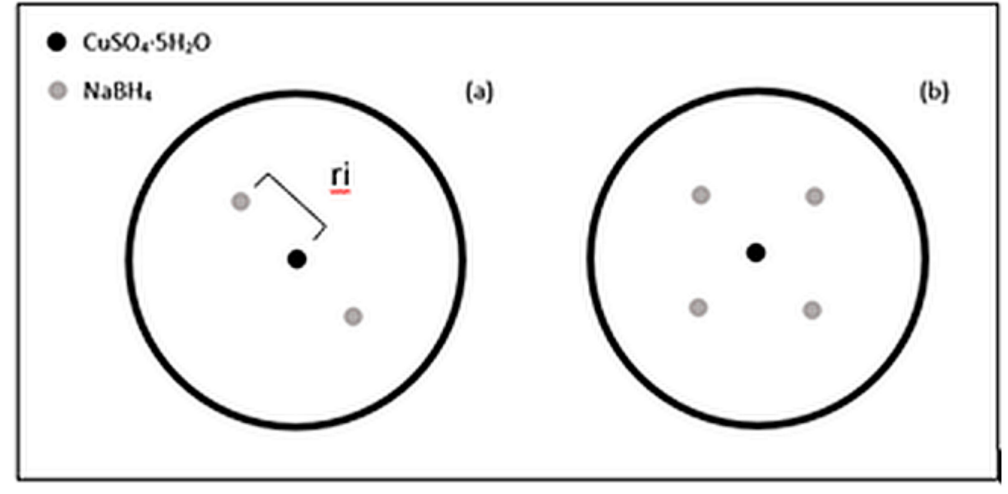

Figure 3. Scheme of the different configuration adopted in this work: (a) for $n f=2 ;(b) n f=4$.

For the experiments concerning changes to the disk velocity $\mathrm{N}$, the relevant results of this experimental campaign are reported in Figure 4 and Table 1. In this case, some operating conditions were fixed, that is, a flow rate from a single feeding point $\mathrm{Qi}$, a distance of the feeding point from the disk center ri, and a number of feeding points nf equal to $151 \mathrm{~h}^{-1}, 2.0 \mathrm{~cm}$ and 2, respectively.

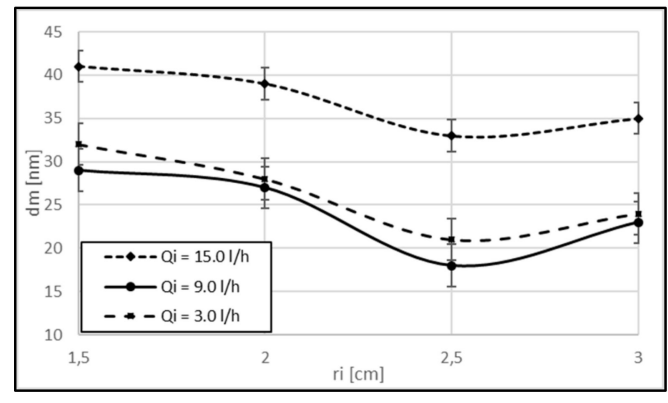

(a)

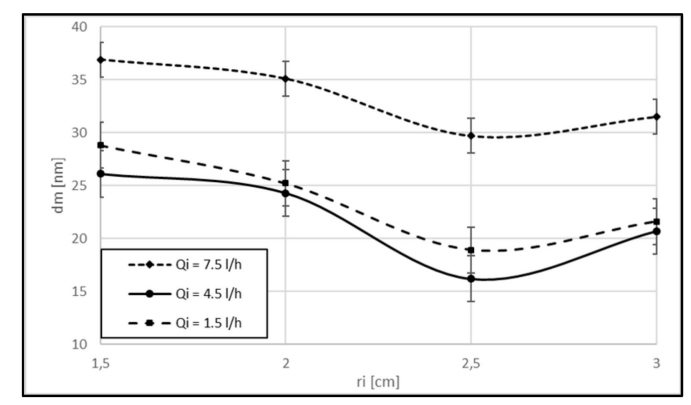

(b)

Figure 4. Average size of copper nanoparticles as a function of the injection distance from the center of the disk using sodium borohydride: $(\mathbf{a})$ for $\mathrm{nf}=2$; $(\mathbf{b}) \mathrm{nf}=4$.

Table 1. Copper nanoparticle size as a function of the disk velocity.

\begin{tabular}{cc}
\hline $\mathbf{N}$ (rpm) & Dm (nm) \\
\hline 500 & 45 \\
1000 & 41 \\
1500 & 39 \\
\hline
\end{tabular}

It appears that a greater value of the disk velocity leads to the production of smaller nanoparticles, with the smallest one produced at the highest limit of rotational speed of the available equipment. As a consequence, the next experiments were all carried out by adopting a disk velocity $\mathrm{N}$ equal to $1500 \mathrm{rpm}$.

The results obtained during the experimental work, and here expressed as the modal size of the produced particles $\mathrm{dm}$, are reported in Figure 4 as a function of Qi, ri, and nf. 
It is possible to observe that for both configurations, the minimum size of produced copper nanoparticles is obtained if a distance of $2.5 \mathrm{~cm}$ from the disc center is used, regardless of the feed flow rate. Moreover, it appears that fixing the feed flow rate equal to $9.01 \mathrm{~h}^{-1}$ and $4.51 \mathrm{~h}^{-1}$ for nf equal to 2 and 4 , respectively, leads to the formation of smallest particles. For nf equal to 2, the produced particles are characterized by a dimension ranging from $18 \mathrm{~nm}$ to $41 \mathrm{~nm}$, with an average size equal to $29 \mathrm{~nm}$. On contrary, in case of using four injection points, the range was reduced to $16-37 \mathrm{~nm}$, with average size of $26 \mathrm{~nm}$. Therefore, a sensible difference on the produced particle size can be observed if two or four injections were in use.

The exact discussion about the resulting dimension of the nanoparticles as a function of the flow rate is complex and involves many aspects, which are out of the scope of this paper. Nevertheless, it should be observed that the film thickness (hundreds of micron) over the disk surface is much smaller than the diameter of the injections points $(\mathrm{mm})$ and the resulting size of the stream entering the available height of the liquid film. In the first step, it appears justified to consider the average Re number only as a function of the disk radius (r), neglecting the vertical distance from the disk surface. In this case, following equation holds [15]:

$$
\operatorname{Re}=\rho U r / \mu
$$

where $\rho$ is the density of the fluid $\left[\mathrm{kg} \cdot \mathrm{m}^{-3}\right] ; \mathrm{r}$ is the radius of the disc of the reactor $[\mathrm{m}]$; $\mathrm{U}$ is the average velocity of the particle in the fluid $\left[\mathrm{m} \cdot \mathrm{s}^{-1}\right] ; \mu$ is the dynamic viscosity $\left[\mathrm{kg} \cdot \mathrm{m}^{-} 1 \cdot \mathrm{s}^{-1}\right]$, the average velocity can be expressed as the ratio between the flow rate $\mathrm{Q}\left[\mathrm{m}^{3} \mathrm{~s}^{-1}\right]$ and the passage area $\mathrm{A}\left[\mathrm{m}^{2}\right]$; and the Reynolds number can be rewritten as:

$$
\operatorname{Re}=\rho \mathrm{Qr} / \mathrm{A} \mu
$$

As a consequence, at constant area values, an increase of the flow rate will lead to higher Re values and thus promote enhanced turbulent processes. Referring to the Kolmogorov scale, it is possible to observe a relationship with the Reynolds number, as follows:

$$
\mathrm{d}_{\mathrm{I}} / \mathrm{d}_{\mathrm{K}}=\mathrm{L}_{\mathrm{I}} / \mathrm{L}_{\mathrm{k}} \cong \mathrm{LU}^{3 / 4} / \mu^{3 / 4} \mathrm{~L}^{1 / 4} \cong \operatorname{Re}^{3 / 4}
$$

where $d_{I}$ is the average diameter of largest scale where the momentum is transported $[m], d_{K}$ is the average diameter of the smallest scale where energy dissipation occurs $[\mathrm{m}], \mathrm{L}_{\mathrm{I}}$ is the characteristic length of the vortices at the different scales $[\mathrm{m}]$, and $\mathrm{L}_{\mathrm{k}}$ is the characteristic length of the vortices at the smallest scale (Kolmogorov scale) [m].

At the end, by considering the average Re number without going into much detail on how this value changes according to the vertical distance from the disk surface, an increase of $Q$ leads to a reduction of the produced particle sizes.

The performed PSD determination on the samples shows that the produced copper nanoparticles are characterized by a unimodal frequency distribution. As ease of an example, Figure 5 shows the PSD of smallest particles reached by adopting the stirred tank reactor (a) and the SDR by using $\mathrm{nf}=2$ (b) and $\mathrm{nf}=4$ (c), respectively. 


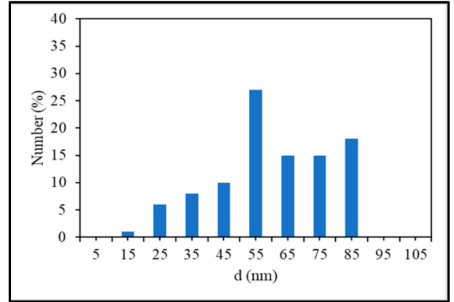

(a)

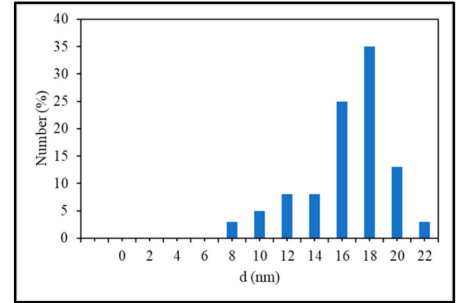

(b)

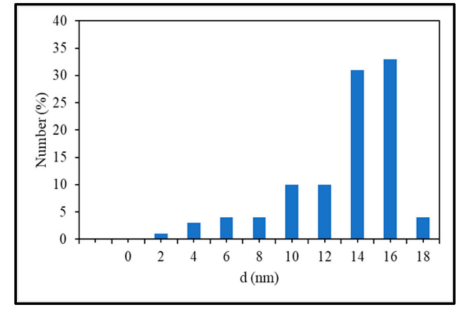

(c)

Figure 5. Results of the dynamic light scattering (DLS) of copper nanoparticles produced with sodium borohydride: (a) in the stirred tank reactor at $1500 \mathrm{rpm}$, (b) by SDR using two injection points; (c) by SDR using four injection points.

\section{Conclusions}

In this work, a suitable production route of copper nanoparticles for industrial application was investigated. The use of an SDR appears to be a promising approach to assist this target. In particular, compared to the stirred tank reactor, the SDR was capable of producing very small copper nanoparticles, characterized by a unimodal and narrow PSD.

The key to success is to identify the proper operating conditions in order to seek the minimization of the nanoparticles size. In this case study, these conditions were identified as a reactant flow rate equal to $4.51 \mathrm{~h}^{-1}$, four feeding points at a distance of $2.0 \mathrm{~cm}$ from the disk center, and a disk velocity equal to $1500 \mathrm{rpm}$. In this case, the outcome of the reaction gave rise to nanoparticles in the range of $10-18 \mathrm{~nm}$, with a modal size of $16 \mathrm{~nm}$.

The tested capacity of the equipment was equal to a continuous production of $5.7 \mathrm{~g} / \mathrm{h}$ of pure copper nanoparticles by a single SDR.

In the future, it might be interesting to study the joint action of the two reducing reagents in the synthesis of Cu-nanoparticles.

Author Contributions: Investigation and experimental work, writing-original draft preparation: A.M.; supervision, data analysis, writing — review and editing: M.S.

Funding: This research received no external funding.

Conflicts of Interest: The authors declare no conflict of interest.

\section{References}

1. Vilardi, G.; Di Palma, L.; Verdone, N. Competitive reaction modelling in aqueous systems: The case of contemporary reduction of dichromates and nitrates by nZVI. Chem. Eng. Trans. 2017, 60, 175-180.

2. Xiong, J.; Wang, Y.; Xue, Q.; Wu, X. Synthesis of highly stable dispersions of nanosized copper particles using L-ascorbic acid. Green Chem. 2011, 13, 900-904. [CrossRef]

3. Vilardi, G. Mathematical modelling of simultaneous nitrate and dissolved oxygen reduction by Cu-nZVI using a bi-component shrinking core model. Powder Technol. 2019, 343, 613-618. [CrossRef]

4. Zhang, Y.; Zhu, P.; Li, G.; Zhao, T.; Fu, X.; Sun, R.; Feng, Z.; Wong, C.P. Facile preparation of monodisperse, impurity-free, and antioxidation copper nanoparticles on a large scale for application in conductive ink. ACS Appl. Mater. Interfaces 2013, 6, 560-567. [CrossRef] [PubMed]

5. Dang, T.M.D.; Le, T.T.T.; Fribourg-Blanc, E.; Dang, M.C. Synthesis and optical properties of copper nanoparticles prepared by a chemical reduction method. Adv. Nat. Sci. Nanosci. Nanotechnol. 2011, 2, 015009. [CrossRef]

6. Badiei, E.; Sangpour, P.; Bagheri, M.; Pazouki, M. Graphene Oxide Antibacterial Sheets: Synthesis and Characterization. Int. J. Eng. Trans. C 2014, 27, 1803-1808.

7. Stoller, M.; Mescia, M.; Peroni, V.C.; Chianese, A. Production of nanoparticles of titanium dioxide by using a spinning disc reactor. Chem. Eng. Trans. 2007, 11, 71-76.

8. De Caprariis, B.; Stoller, M.; Chianesea, A.; Verdone, N. CFD model of a spinning disk reactor for nanoparticle production. Chem. Eng. 2015, 43, 757-762. [CrossRef] 
9. Stoller, M.; Di Palma, L.; Vuppala, S.; Verdone, N.; Vilardi, G. Process intensification techniques for the production of nano-and submicronic particles for food and medical applications. Curr. Pharm. Des. 2018, 24, 2329-2338. [CrossRef] [PubMed]

10. Ochando Pulido, J.M.; Stoller, M. Kinetics and boundary flux optimization of integrated photocatalysis and ultrafiltration process for two-phase vegetation and olive washing wastewaters treatment. Chem. Eng. J. 2015, 279, 387-395. [CrossRef]

11. Vilardi, G. Bimetallic nZVI-induced chemical denitrification modelling using the shrinking core model. Chem. Eng. Trans. 2018, 70, 235-240.

12. Stoller, M.; Miranda, L.; Chianese, A. Optimal feed location in a spinning disc reactor for the production of TiO2 nanoparticles. Chem. Eng. Trans. 2009, 17, 993-998.

13. Vilardi, G.; Stoller, M.; Verdone, N.; Di Palma, L. Production of nano Zero Valent Iron particles by means of a spinning disk reactor. Chem. Eng. Trans. 2017, 57, 751-756.

14. Ochando-Pulido, J.M.; Stoller, M.; Di Palma, L.; Martínez-Férez, A.; Vilardi, G. Spinning Disk Reactor Technology in Photocatalysis: Nanostructured Catalysts Intensified Production and Applications. Nanophotocatal. Environ. Appl. 2019, 29, 303-333. [CrossRef]

15. Vilardi, G.; Stoller, M.; Di Palma, L.; Verdone, N. CFD Model of Agitated Vessel for the Removal of Cr(vi) by Nano-hematite Particles. Chem. Eng. Trans. 2019, 73, 157-162.

(C) 2019 by the authors. Licensee MDPI, Basel, Switzerland. This article is an open access article distributed under the terms and conditions of the Creative Commons Attribution (CC BY) license (http://creativecommons.org/licenses/by/4.0/). 